\title{
LTE IDLE MODE OPTIMIZATION IMPROVING END USER EXPERIENCES
}

\author{
Kwangrok Chang, Ragil Putro Wicaksono, Seiji Kunishige, Noriteru Takagaki \\ MOTiV Research Co. Ltd., Tokyo Japan \\ \{kwangrok.chang, ragil.wicaksono, seiji.kunishige, noriteru.takagaki\}@motiv-research.com
}

\begin{abstract}
This paper presents the methodology of defining the measurement trigger conditions and evaluation conditions of LTE cell reselection for release 9 capable UEs. In the proposal of cell reselection parameters, the RSRQ measurement quantity is considered to account for the increase of packet traffic in LTE networks. In addition, the proposal is designed to improve the end user experiences such the LTE camp-on ratio, the avoidance of unnecessary cell reselection measurements causing the battery drain and the ping-pong effect between LTE and WCDMA interRAT cell reselection or redirection. The cell reselection parameter proposal is made based on the field test data collected in Tokyo metropolitan area.
\end{abstract}

Keywords — LTE release 9, cell reselection, RSRQ, idle mode parameters

\section{INTRODUCTION}

The UEs capable of 3GPP Release9 specification started emerging in the mobile phone markets in the world from 2013 and eventually Apples' iPhone $5 \mathrm{~s} / 5 \mathrm{c}$ and other latest smartphones have been also released stirring up lots of UE subscribers' desire to purchase the LTE UEs. Even if the operators have not experienced high packet data traffic brought by the smart phones yet and do not have quantitatively measurable record to tell how much the packet traffic will increase by the LTE UEs, they can down to earth anticipate that the packet traffic volume in LTE RAN will arise significantly and shortly, which will end up with serious RSRQ deterioration in RF coverage perspective. In this case, the Release8 idle mode parameters for determining UE's cell reselection behaviour [1], [2], which adopts only RSRP of the serving cell and the target cells, won't be good enough in the high traffic network scenario and the operators will need the provision of Release 9 idle mode parameters to accommodate the RSRQ measurement quantity into idle mode cell reselection [3]. It is inferable that some operators have already revised their idle mode parameter settings in the preparation of release 9 LTE UE launch.

\section{LTE IDLE MODE OPTIMIZATION FOR END USER EXPERIENCES}

As in the WCDMA network where EcNo gets lower resulted from the increase of own cell traffic and/or other cell traffic, RSRQ in LTE network is also degraded when the traffic in the own cell and/or other cell grows. If UE's cell reselection is performed only according to RSRP but not to RSRQ, which is Release 8 version, UE won't be able to perform the cell reselection to a superior LTE target layer cells even if the serving cell's RSRQ went to a significantly low level due to high traffic. As a result, the end user will experience poor packet performance if RRC connection is started.

RSRQ driven cell reselection becomes crucial in case there is a possibility that the LTE traffic would not be equally distributed over the multiple LTE layers. For example, if an operator is running multiple LTE frequency bands where the eNB's antenna for each LTE frequency band is located in different spots resulting in each eNB posing different RF coverage footprints or even if the eNB for each frequency band forms the similar RF coverage layout, RSRQs won't be the similar level in case the eNB vendors do not support traffic load balancing feature over multiple LTE bands. Also if the eNB vendors are different for each frequency band, the load balancing between the multi-vendor are not generally supported and as a result, RSRQ of the different frequency bands could be far different from each other even if RSRP levels are similar.

Cell reselection is performed according to the system information parameters broadcasted in SIB3, SIB5, SIB6 of LTE and SIB19 of WCDMA. The new parameters additionally provided in Release 9 in the SIBs are summarized TABLE 1 [3],[4].

TABLE 1. NEW LTE IDLE MODE PARAMETERS IN RELEASE 9

\begin{tabular}{|c|c|}
\hline SIB Type & Parameters \\
\hline SIB3 & $\begin{array}{l}\text { s-IntraSearchP-r9 } \\
\text { s-IntraSearchQ-r9 } \\
\text { s-NonIntraSearchP-r9 } \\
\text { s-NonIntraSearchQ-r9 } \\
\text { q-QualMin-r9 } \\
\text { threshServingLowQ-r9 }\end{array}$ \\
\hline SIB5 & $\begin{array}{l}\text { q-QualMin-r9 } \\
\text { threshX-HighQ-r9 } \\
\text { threshX-LowQ-r9 }\end{array}$ \\
\hline SIB6 & $\begin{array}{l}\text { threshX-HighQ-r9 } \\
\text { threshX-LowQ-r9 }\end{array}$ \\
\hline $\begin{array}{c}\text { SIB19 } \\
\text { (WCDMA) }\end{array}$ & $\begin{array}{l}\text { qualMinEUTRA } \\
\text { threshXhigh2 } \\
\text { threshXlow2 }\end{array}$ \\
\hline
\end{tabular}

It is aimed in this paper to propose the cell reselection parameters taking into account the traffic load increase (RSRQ measurement quantity) for LTE network. The target UE model 
of the new cell reselection parameters is the Release 9 capable UEs. In the new cell reselection parameter proposal, the RSRQ threshold for the measurement trigger and the evaluation condition are investigated. The test area where the new cell reselection parameters are developed is dense urban areas in Tokyo with high packet traffic.

\section{ENd USER's IdLe MOde Performance IN LTE NETWORK}

The LTE network operator's objective for idle mode is to have the LTE capable UEs hold the LTE coverage as long as possible showing ' $4 \mathrm{G}$ ' or 'LTE' symbol on the mobile's screen. The assessment of how long or how high chance of camping on the LTE cells rather than $3 \mathrm{G}$ cells can be articulated by a performance indicator called 'LTE camp-on ratio', which is the ratio of the LTE data samples against the $3 \mathrm{G}$ data samples collected by UE. The LTE camp-on ratio is determined by the parameters relevant to the S-criteria and the evaluation condition for LTE to WCDMA cell reselection. Between the two conditions, the evaluation condition is crucial to influence the LTE camp-on ratio as the evaluation condition starts earlier than S-criteria by the value of Thresh $h_{\text {serving,low. }}$.

As the $1^{\text {st }}$ step of defining the UE's measurement trigger for the cell reselection, it is considered the end user idle mode performance, which is interpreted as the number of LTE signal bars displayed on the UE's screen. Although the relation between the number of signal bars and the packet call performances may need to be studied separately however, in general if the number of signal bars displayed is ' 3 ' or more, the LTE coverage can be considered acceptable or satisfying level from end user's perspective. Then, it is worthy to study how the UE's signal bars are determined upon LTE coverage condition. iPhone5 was used for this investigation.

In order to study the relation between the number of the signal bars and the actual RF coverage - RSRP and RSRQ, the field test using iPhone5 and RF scanner was performed. The RSRP and RSRQ measured by the scanner and the number of signal bars data collected from the iPhone5 at the same time stamp are compared for the same PCI that the iPhone5 was camped on in idle mode.

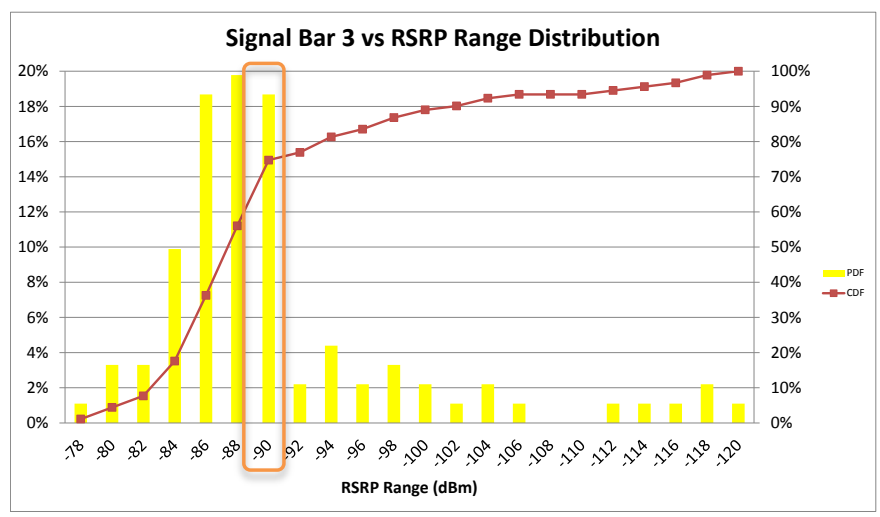

Figure 1. RSRP PDF and CDF for signal bar 3
Figure 1. shows that RSRP $=-90 \mathrm{dBm}$ is apparent threshold by which the signal bar 3 is determined in iPhone5. It can be said that serving cell's RSRP shall be greater than or equal to $90 \mathrm{dBm}$ to meet the end user's RF coverage satisfaction, which is signal bar 3 on screen.

$$
R S R P_{\text {serving }} \geq-90 \mathrm{dBm} \text { for signal bar } 3
$$

\section{IV.DEFINING RSRP AND RSRQ THRESHOLDS FOR MEASUREMENT TRIGGER}

UE's measurement for intra-frequency and inter-frequency cell reselection shall be triggered at least before reaching RSRP $=-90 \mathrm{dBm}$. In general, in the areas nearby the serving cell's antenna site, the RSRP level must be good enough not requiring the cell reselection to the neighboring cells and also the neighbor cell's RSRP condition is not at the comparable level to the serving cell's. At the cell edge or cell border areas, the serving cell's RSRP and RSRQ must be deteriorated due to the propagation loss and noise and other cell interferences. It can be re-written that the cell reselection measurement trigger at the serving cell's edge needs to be triggered with high certainty that the serving cell's RSRP will not fall below $-90 \mathrm{dBm}$. The definition of serving cell edge is expressed as follow in this paper.

$$
\Delta R S R P=R S R P_{\text {serving }}-R S R P_{\text {neighbor }}<3 d B
$$

It is noted from Figure 2. that the ratio of cell edge, defined by $\triangle \mathrm{RSRP}$, against the overall data samples is slightly less than $20 \%$ at RSRQ $=-10 \mathrm{~dB}$. However, when the RSRQ is $9 \mathrm{~dB}$ or higher, the ratio of cell edge sample is negligible and in adverse, the inner area samples where no suitable neighbor cell for cell reselection can be found approaches almost $100 \%$.

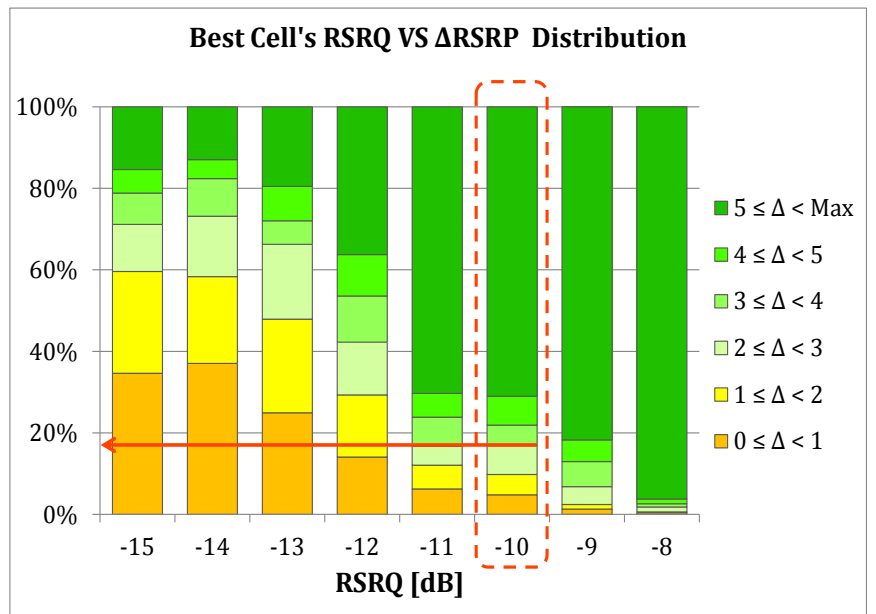

Figure 2. Ratio of various $\Delta$ RSRP for the Best cell's RSRQ

It explains that if RSRQ $=-9 \mathrm{~dB}$ or higher value is used for the measurement trigger for the cell reselection, nearly $100 \%$ of locations within the cell's coverage area is exposed to UE's measurement trigger even though there won't be suitable neighbor cells available for the cell reselection. The RSRQ, - 
$9 \mathrm{~dB}$ causes unnecessary cell reselection triggers to occur too often and therefore, it is desirable to use $-10 \mathrm{~dB}$ for the RSRQ threshold for Release 9 UE's measurement trigger.

$$
R S R Q_{\text {serving }} \leq-10 \mathrm{~dB}
$$

The next step is to define the measurement trigger threshold of RSRP for release 9 UE. The lower bound of RSRP has been defined as $-90 \mathrm{dBm}$. The upper bound for RSRP, shall be determined in a way that UE can avoid the unnecessary measurements for intra-frequency and inter-frequency neighbors. The PDF distribution of RSRP and RSRQ measured by RF scanner is presented in Figure 3. .

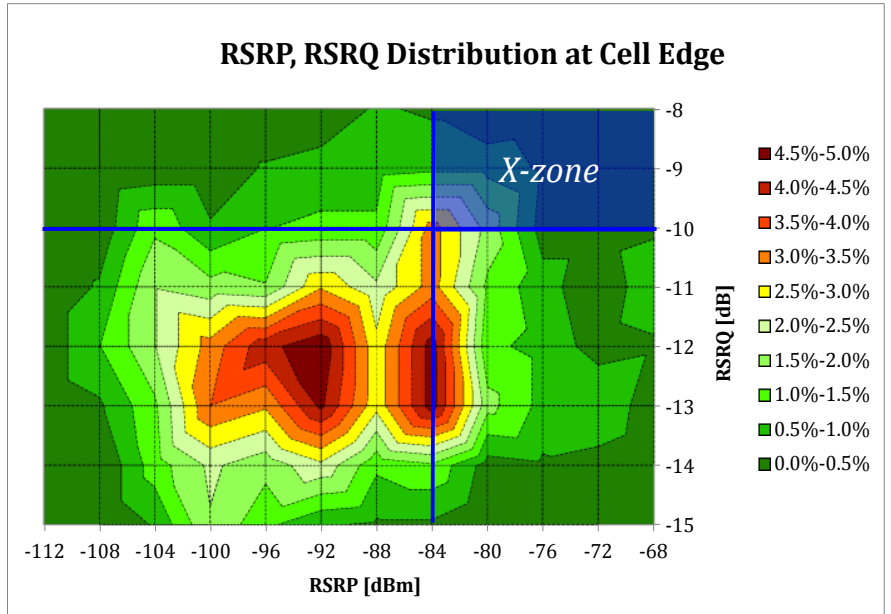

Figure 3. PDF of RSRP and RSRQ in a dense urban area LTE network

The ' $X$-zone', shown in Figure 3., is the data samples where the cell reselection measurement won't be triggered for the given RSRP and RSRQ thresholds. (e. g. RSRP and RSRQ threshold was given as $-84 \mathrm{dBm}$ and $-10 \mathrm{~dB}$ respectively)

From this network measurement data, it is calculated in TABLE 2 how much ratio, the cell reselection measurement will occur at the cell edge and inner areas within the cell's whole coverage when the different RSRP thresholds were used. In this calculations, RSRQ threshold is fixed to $-10 \mathrm{~dB}$.

TABLE 2. INTRA-FREQUENCY MEASUREMENT RATIO AT THE CELL EDGE AND INNER AREAS FOR DIFFERENT RSRP

\begin{tabular}{|c|c|}
\hline RSRP $[\mathrm{dBm}]$ & Intra Ncell Measurement Area (inner area) \\
\hline-80 & $75 \%$ \\
\hline-84 & $65 \%$ \\
\hline-88 & $60 \%$ \\
\hline-92 & $55 \%$ \\
\hline
\end{tabular}

The higher the measurement ratio at the cell edge while the lower measurement ratio in inner area, the better cell reselection efficiency we aim to have. When $\mathrm{RSRP}=-84 \mathrm{dBm}$ is selected for the trigger threshold, we can avoid unnecessary measurements in the inner area while having $92 \%$ of cell edge area be subject to the measurement trigger.
Therefore, it is recommended to set the thresholds of RSRP for the release 9 UE's intra-frequency cell reselection measurement trigger as follow:

$$
R S R P_{\text {serving }} \leq-84 \mathrm{dBm}
$$

The cell reselection measurement for the inter-frequency neighbors of which priority is equal to or lower than the serving LTE layer, needs to be triggered marginally later than the intra-frequency measurement by $2 \mathrm{~dB}$. Therefore it is suggested to define the inter-frequency cell reselection measurement as below:

$$
R S R P_{\text {serving }} \leq-86 \mathrm{dBm} \text { or } R S R Q_{\text {serving }} \leq-12 \mathrm{~dB}
$$

\section{DEFINING RSRP AND RSRQ THRESHOLDS FOR EVALUATION CONDITION}

When the target inter-frequency LTE layer or target interRAT 3G layer is lower priority than the serving LTE layer, there are three variants of cell reselection evaluation conditions for the release 9 capable UE. For Release 9 UE with threshServingLow $Q$ provided:

$$
\begin{aligned}
& R S R Q_{\text {serving }}<Q_{\text {qualmin }}+\text { Thresh }_{\text {Serving,LowQ }} \\
& A N D R S R Q_{\text {neighbor }}>Q_{\text {qualmin }}+\text { Thresh }_{X, L o w Q} \\
& R S R Q_{\text {serving }}<Q_{\text {qualmin }}+\text { Thresh }_{\text {Serving,LowQ }} \\
& A N D E c N o_{\text {neighbor }}>Q_{\text {qualminUTRA }}+\text { Thresh }_{X, \text { LowQ }} \\
& \text {, where } Q_{\text {qualminoffset }} \text { is assumed to be ' } 0 \text { '. }
\end{aligned}
$$

For Release 9 UE without threshServingLowQ provided:

$$
\begin{aligned}
& R S R P_{\text {serving }}<Q_{\text {rxlevmin }}+\text { Thresh }_{\text {Serving,LowP }} \\
& \text { AND } R S R P_{\text {neighbor }}>Q_{\text {rxlevmin }}+\text { Thresh }_{X, L o w P} \\
& R S R P_{\text {serving }}<Q_{\text {rxlevmin }}+\text { Thresh }_{\text {Serving,LowP }} \\
& \text { AND } R S C P_{\text {neighbor }}>Q_{\text {rxlevminuTRA }}+\text { Thresh }_{X, L o w P} \text {, } \\
& \text {, where } Q_{\text {rxlevminoffset }} \text { is assumed to be ' } 0 \text { '. }
\end{aligned}
$$

If the inter-frequency LTE layer has the same priority than the serving LTE layer does, the evaluation condition is given as below:

$$
R S R P_{\text {neighbor }}+Q_{\text {offset }}>R S R P_{\text {serving }}+\text { Qhyst }_{\text {s }}
$$

It is known from all the evaluation conditions expressed above that RSRQ measurement quantity is used for release 9 UE with threshServingLow $Q$ parameter provided in SIB3. Since it is aimed to develop an inter-frequency cell reselection evaluation condition taking into account the traffic load not only the RF coverage, RSRQ based evaluation condition needs to be investigated.

The RSRQ evaluation threshold for the serving LTE cell, ' $Q_{\text {qualmin }}+$ Thresh $_{\text {Serving,LowQ }}$ ' defines the lower bound of LTE coverage that if RSRQ gets worse than this value, UE will perform inter-RAT cell reselection to WCDMA (assumed that WCDMA coverage is always higher than the lower bound 
of WCDMA coverage of evaluation condition). In order to determine the RSRQ evaluation threshold, it is noted that there is another lower bound of RSRQ in spite it is not in idle more but in the RRC connected mode by which EUTRAN can release the RRC connection by force and to redirect the UE to WCDMA if RSRQ gets lower than this value. This parameter in connected mode is called a2-threshold [5]. The parameter value of a2-threshold provided in the rrcConnectionReconfiguration message governs UE's measurement report of event A2.

When RSRQ is worse than a2-threshold due to high traffic load or high interference, it is reckoned that LTE throughput performance is no more superior to 3G's so that the network configures in EUTRAN to redirect the UE to $3 \mathrm{G}$ network. It means that even if the evaluation threshold, ' $Q_{\text {qualmin }}+$

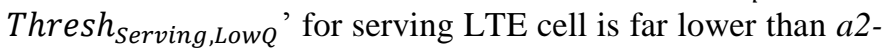
threshold, in other words, UE did not make cell reselection to WCDMA even RSRQ is far lower than a2-threshold, the UE will be redirected to $3 \mathrm{G}$ once the UE enters the RRC connected mode. Therefore it is desirable to set the same RSRQ value for the evaluation threshold, ' $Q_{\text {qualmin }}+$

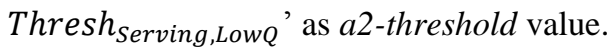

The next step is to determine the value of Thresh $_{X, L o w Q}$, which is used in the target cell's evaluation condition. This parameter is also used in both inter-frequency and inter-RAT cell reselection evaluations. The parameter, Thresh ${ }_{X, L o w Q}$ is a sort of hysteresis to avoid the ping-pong that UE comes back to the old serving cell's layer soon after the inter-frequency LTE cell reselection was made. The parameter, Thres $h_{X, L o w Q}$ needs to be defined so as to be less sensitive to the instantaneous fluctuation of RSRQ.

The stationary RSRQ measurement was performed at a station of Tokyo city to grab how much RSRQ level fluctuates. The measurement result is presented in RSRP and RSRQ fluctuation range in urban stationary test.

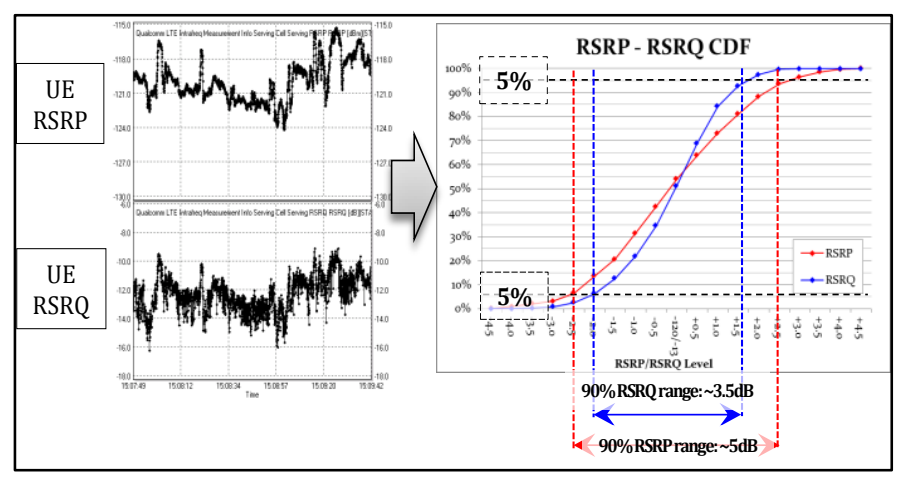

Figure 4. RSRP and RSRQ fluctuation range in urban stationary test

It is revealed that $90 \%$ of RSRQ samples centred on the median resides within $3.5 \mathrm{~dB}$ fluctuation range. RSRP's fluctuation range is $5 \mathrm{~dB}$ as reference information.

Taking into account 3dB RSRQ fluctuation range, the target layer's RSRQ shall be higher than serving layer's RSRQ by $3 \mathrm{~dB}$ or $4 \mathrm{~dB} .4 \mathrm{~dB}$ of Thresh $_{X, \text { LowQ }}$ could be too high to satisfy the evaluation condition for the target cell. Therefore, it is recommended to use $3 \mathrm{~dB}$ and as a result the parameter value of Thresh $h_{X, \text { LowQ }}$ is defined as:

$$
\text { Thresh }_{X, \text { Low Q }}=\text { Thresh }_{\text {Serving,LowQ }}+3 d B
$$

By deciding the parameter values of Thresh Serving,LowQ $_{\text {, }}$ and Thresh $_{X, L o w Q}$, the evaluation condition utilizing RSRQ is completed.

\section{CONCLUSIONS}

The cell reselection parameters for release 9 capable UE proposed in this paper can be various depending on the operator's strategy of LTE network deployment and the absolute layer priorities between the LTE layers and WCDMA. Nevertheless, the presented procedure and the methodology used in this paper can be reused in any LTE networks with a certain degree of customizations. Using the proposed values, $8 \%$ of unnecessary cell reselection measurement trigger could be avoided and $22 \%$ of the end user perception of LTE coverage during the idle mode could be improved in Tokyo metropolitan area.

\section{REFERENCE}

[1] 3GPP TS36.304 v8.3.0, E-UTRA UE procedures in idle mode (Release 8)

[2] 3GPP TS36.133 v8.3.0, E-UTRA Requirements for support of radio resource management (Release 8)

[3] 3GPP TS36.304 v9.8.0, E-UTRA UE procedures in idle mode (Release 9)

[4] 3GPP TS25.304 v9.8.0, UE procedures in idle mode and procedures for cell reselection in connected mode (Release 9)

[5] 3GPP TS36.331 v9.8.0, E-UTRA Radio Resource Control (RRC) Protocol specification (Release 9)

[6] 3GPP TS25.304 v8.12.0, UE procedures in idle mode and procedures for cell reselection in connected mode (Release 8)

[7] 3GPP TS25.133 v8.3.0, Requirements for support of radio resource management (FDD) (Release 8)

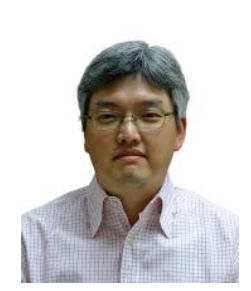

Kwangrok Chang (B.E.'93-M.E.'95-Ph.D.'98) received the degrees of Master and $\mathrm{Ph} . \mathrm{D}$ in Electronic and Electrical Engineering from POSTECH in 1995 and 1998, respectively. Since 2000, he has been with Nokia Siemens Networks Ltd. (NSN), where he led the Network Planning and Optimization Group of Japan and Korea as the NPO head. He left NSN in 2011 and co-founded MOTiV Research Co., Ltd with Seiji Kunishige aiming for providing communication service providers and system vendors with the mobile network planning strategy and optimization services and the development of the advanced network performance enhancement methodology based on the big network data. His present interests are the enhancement of end user performances in high speed data networks, such as HSPA and LTE and the innovation of network performance data collection and its processing.

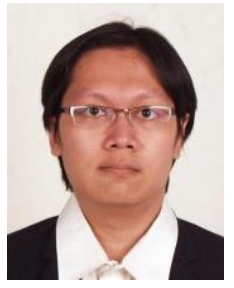

Ragil Putro Wicaksono (B.Eng'06-M.Eng'11) had been working with NSN as network planning engineer for 2.5 years before received the degrees of Master in Electronic and Electrical Engineering from Tokyo Institute of Technology (東京工業大学), Japan, on September, 2011. Afterwards he works in MOTiV Research Co. Ltd., as a cellular network consultant. His research interest are mainly related with mobility and 
radio resource management in cellular network (WCDMA, LTE, and LTE-A).

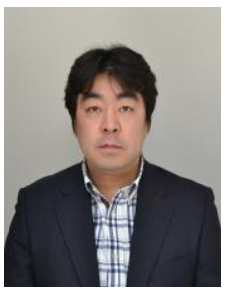

Seiji Kunishige (B.Eng'97) had worked at Couei as customer service engineer for first launch of CDMA network(CDMA-one) in Japan for 4 years after graduated Kansai University. He has worked at Nokia Siemens Networks since 2001 and joined Network Planning and Optimization team. He led technical consulting team of WCDMA/HSPA networks as Solution Architect manager. He co-founded MOTiV Research Co., Ltd with Kwangrok Chang as CTO at 2011 and he is currently in charge of the general management of technical issues.

Noriteru Takagaki had been with NSN for a decade mainly focusing on network key performance indicator analysis and its data processing to identify the root causes of the abnormal network call performances. In 2011, he joined MOTiV Research Co., Ltd. as the field test team manager maintaining the quality of field tests and improving the efficiency of the network data collections. 